\title{
Efeitos do Pilates na qualidade do sono em adultos e idosos: uma revisão sistemática
}

\author{
Effects of Pilates on sleep quality in adults and elderly: a systematic review
}

\section{AUTORES \\ Ozeas de Lima de Lins-Filho ${ }^{1}$ (D) Georgia Kelly Ferreira de Queiroz ${ }^{2}$ (iD) Jefferson Felipe Julião Santos ${ }^{2}$ (D) Marcos André Moura dos Santos ${ }^{3}$ Luciano Machado Ferreira Tenório de Oliveira ${ }^{4}$ (1) Breno Quintella Farah ${ }^{5}$ \\ 1 Universidade de Pernambuco, Programa de Pós-Graduação em Ciências da Saúde, Recife, Pernambuco, Brasil. \\ 2 Grupo de Pesquisa em Saúde e Esporte do Centro Universitário Tabosa de Almeida ASCES/UNITA, Caruaru, Pernambuco, Brasil. \\ 3 Universidade de Pernambuco, Programa Associado de Pós-Graduação em Educação Física UPE/UFPB, Recife, Pernambuco, Brasil. 4 Centro Acadêmico de Vitória da Universidade Federal Rural de Pernambuco, Vitória de Santo Antão, Pernambuco, Brasil. \\ 5 Departamento de Educacão Física da \\ Universidade Federal Rural de Pernambuco, Recife, Pernambuco, Brasil.}

\section{CONTATO}

Dr. Breno Quintella Farah

breno.farab@ufrpe.br

Departamento de Educação Física, Universidade Federal Rural de Pernambuco

Rua Dom, R. Manuel de Medeiros, s/n -

Dois Irmãos, Recife, Pernambuco.

CEP: 52171-900.

DOI

$10.12820 /$ rbafs. $24 \mathrm{e} 0080$

\begin{abstract}
RESUMO
O objetivo do presente estudo foi sintetizar, por meio de uma revisão sistemática, os efeitos do Pilates na qualidade de sono em adultos e idosos. Para tanto, recorreu-se à revisão sistemática de ensaios clínicos que analisaram os efeitos do Pilates na qualidade do sono, publicados em periódicos indexados na base de dados do PubMed/Medline, LILACS (Literatura Latino-Americana e do Caribe em Ciências da Saúde), SciELO (Scientific Electronic Library Online) e SCOPUS publicados até 2019. Foram incluídos oito estudos nesta revisão, totalizando 608 participantes de 18 a 64 anos de idade. Dentre os estudos, três incluíram idosos na amostra. Os protocolos dos programas variaram de 4 a 16 semanas, com 2 a 3 sessões semanais, com duração média de 120 a 180 minutos de atividade por semana. A quantidade de exercícios variou de 13 a 15 exercícios, realizados em 1 ou 2 séries, com 8 a 12 repetições, trabalhando o controle da respiração. Todos os estudos utilizaram o Mat Pilates como forma de intervenção e avaliaram a qualidade do sono com questionário Pittsburgh Sleep Quality Index (PSQI) ou da Escala de Sonolência de Epworth (ESS). Foi observado nestes estudos uma melhora do PSQI com base no aumento da eficiência e qualidade geral do sono e redução na latência do sono, bem como uma redução no escore da ESS após as intervenções. Os resultados reportados indicaram que o programa de Mat Pilates promoveu melhoria na qualidade do sono de adultos e idosos.
\end{abstract}

Palavras-chave: Técnicas de exercício e de movimento; Distúrbios do início e da manutenção do sono; Sono.

\begin{abstract}
The aim of this study was to synthesize, through a systematic review, the effects of Pilates on sleep quality in adults and elderly. Therefore, a systematic review of clinical trials analyzing the effects of Pilates on sleep quality, published in journals indexed in the PubMed/Medline database, LILACS (Latin American and Caribbean Center on Health Sciences), SciELO (Scientific Electronic Library Online) and SCOPUS published until 2019 was performed. Eight studies were included in this review, with total sample size of 608 participants aged from 18 to 64 years. Among the studies, three included the elderly in the sample. Program protocols ranged from 4 to 16 weeks, 2 to 3 sessions, average duration of 120 to 180 minutes per week. The number of exercises ranged from 13 to 15 exercises, performed in 1 or 2 sets, 8 to 12 repetitions, with breathing control. All studies used the Mat Pilates as intervention and assessed sleep quality using the Pittsburgh Sleep Quality Index (PSQI) or the Epworth Sleepiness Scale (ESS). It was observed an improvement in the $P S Q I$ score through increased sleep efficiency and overall quality and reduced sleep latency, and a reduction in ESS score after interventions. The reported results indicated that the Mat Pilates program improved sleep quality in adults and elderly.
\end{abstract}

Keywords: Exercise movement techniques; Sleep initiation and maintenance Disorders; Sleep.

\section{(cc) BY-NC-SA}

Este obra está licenciado com uma Licença

\section{Introdução}

Atualmente, um terço da população adulta em todo o mundo é afetada por diversos distúrbios do sono ${ }^{1}$. No Brasil, estima-se cerca de dez a vinte milhões de pessoas apresentando problemas relacionados ao sono ${ }^{2}$. Esses dados, são preocupantes, do ponto de vista da saúde pública, uma vez que a qualidade do sono está diretamente associada a doenças crônicas não transmissíveis, como: hipertensão arterial, obesidade e síndrome metabólica ${ }^{3-5}$, além da associação com uma pior qualidade de vida ${ }^{6}$.

Em detrimento deste cenário e do o possível papel regulador do exercício físico, algumas investigações têm sido descritas sobre o impacto positivo do exercício físi- 
co na qualidade do sono ${ }^{7-9}$. Dentre os diferentes tipos de exercício o Pilates tem se mostrado eficaz na melhoria da qualidade do sono ${ }^{10,11}$. Caldwell et al. ${ }^{10}$ observaram que a realização de 15 semanas de Pilates melhorou a qualidade do sono de jovens universitários. Da mesma maneira, Asharafinia et al. ${ }^{11}$, ao submeterem mulheres pós-parto, a oito semanas de Pilates, observaram melhoria na qualidade do sono. Aspecto também descrito e observado em outros estudos mais recentemente ${ }^{12-14}$.

Contudo, embora esses resultados sejam promissores e forneçam indicativos da eficácia do Pilates na melhoria da qualidade do sono, não há padronização na aplicação dos protocolos previamente publicados visto que há grande quantidade de variáveis a serem moduladas para obter sucesso com a intervenção, ${ }^{15}$ bem como não está claro quais componentes da qualidade do sono são modificados após intervenção. Por exemplo, estudo prévio ${ }^{16}$ verificou aumento da qualidade do sono em 31 idosas após 16 semanas de Pilates através de redução na latência do sono e no uso de medicações para dormir. Por outro lado, Ahmadinezhad et al. ${ }^{12}$ observaram aumento na qualidade do sono pelo aumento na eficiência do sono e redução na latência e na disfunção diurna em 36 mulheres com um protocolo com duração de apenas 6 semanas. Ademais, a heterogeneidade quanto a população analisada entre os estudos (mulheres pós-menopausa, idosos, universitários, pacientes com doenças crônicas e adultos jovens sedentários) e a ausência de revisão sistemática sobre a temática, impossibilita conclusões a respeito dos efeitos do Pilates na qualidade do sono.

Nesse sentido, esta revisão sistemática permitirá uma visão global dos estudos que analisaram os efeitos do $\mathrm{Pi}^{-}$ lates na qualidade do sono de adultos e idosos, no que tange os protocolos utilizados, haja vista as inúmeras maneiras de realizar o Pilates, bem como permitirá a análise da qualidade metodológica dos estudos, possibilitando uma visão mais criteriosa dos estudos da literatura. Em conjunto, essas respostas poderão auxiliar profissionais de saúde a desenvolver intervenções eficazes na melhoria da qualidade do sono. Portanto, o objetivo deste estudo é sintetizar, por meio de uma revisão sistemática, os efeitos do Pilates na qualidade de sono em adultos e idosos.

\section{Métodos}

Essa revisão sistemática foi realizada de acordo com Preferred Reporting Items of Systematic Reviews and Meta-Analyses (PRISMA) ${ }^{17}$ e foi registrada no Prospectively Registered Systematic Reviews (PROSPERO) CRD42019119985.

Realizou-se uma revisão sistemática de ensaios clínicos publicados em periódicos indexados na base de dados eletrônica PubMed/Medline (National Library of Medicine - NLM), LILACS (Literatura Latino-Americana e do Caribe em Ciências da Saúde), SciELO (Scientific Electronic Library Online) e SCOPUS que continham análises referente ao efeito do Pilates na qualidade do sono de indivíduos com idade maior ou igual a 18 anos, publicados até maio de 2019. Os descritores utilizados na pesquisa eletrônica foram definidos mediante pesquisa prévia em Medical Subject Headings (MeSH) e separados em três blocos (população, método Pilates e qualidade do sono). Para população os seguintes descritores foram utilizados: "adult", "middle age", "aging", "elderly" e "aged"; para o método Pilates: "Pilates based exercises", "Pilates training" e "exercise movement techniques"; e para qualidade do sono: "Insomnia", sleep initiation and maintenance disorders", "sleep Quality", "sleep disturbance” e "sleep”. Os descritores foram combinados utilizando os operadores booleanos, conforme exibido na Figura 1.

A busca eletrônica nas bases de dados foi realiza-

\begin{tabular}{|c|}
\hline População \\
\hline Mesh \\
\hline Adult \\
OR \\
Middle Age \\
OR \\
Aging \\
OR \\
Elderly \\
OR \\
Aged \\
\end{tabular}

Figura 1 - Descritores utilizados na busca
AND

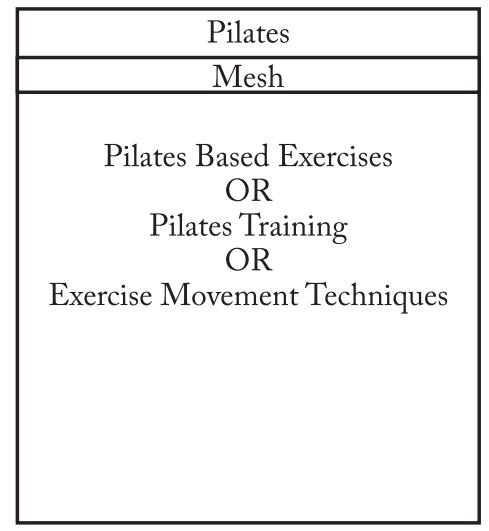

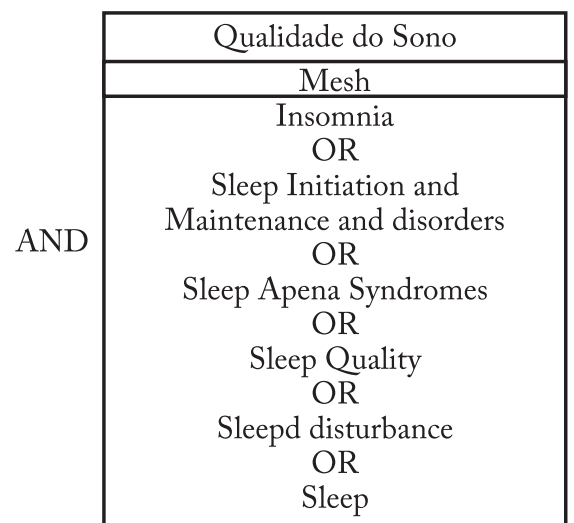


da com a combinação dos descritores e a aplicação dos respectivos limites. Foram incluídos apenas os ensaios clínicos sem limite de anos ou idioma (Etapa 1). A etapa 2 consistiu em leitura dos títulos e dos resumos das publicações. Foram incluídos apenas os estudos que preencheram os seguintes critérios: intervenção com Pilates, avaliação da qualidade do sono geral, amostra composta exclusivamente por adultos e idosos e sem restrição de sexo. $\mathrm{Na}$ etapa 3, realizou-se a leitura dos artigos na íntegra e a extração dos dados. Nessa etapa, foram extraídos os dados sobre: característica da amostra, o protocolo de exercício e protocolo de avalição do sono.

Todas as etapas (busca eletrônica na base de dados, seleção e avaliação dos artigos potenciais, extração dos dados dos estudos e análise da qualidade dos estudos) foram realizadas por dois pesquisadores independentes. Os resultados de cada etapa foram comparados por um terceiro avaliador a fim de verificar a concordância entre os pares. $\mathrm{Na}$ eventualidade de divergências, um terceiro orientador foi responsável pela análise final. A análise da qualidade dos estudos foi feita por meio da escala PEDro ${ }^{18}$, especifica para estudos experimentais. Essa escala é composta por 11 questões, que visa avaliar as validades interna e externa dos estudos sendo atribuídas notas que variam de zero (mínima) a dez (máxima). A classificação da escala PEDro aloca três pontos aos métodos de cegamento utilizados, dois pontos aos procedimentos de randomização, dois pontos atribuídos os relatos apropriados de dados e um ponto para cada análise de dado e adequação ao acompanhamento. Uma pontuação maior do que seis indica qualidade do artigo de moderada a alta qualidade.

\section{Resultados}

$\mathrm{Na}$ Figura 2 é apresentado o fluxograma dos estudos incluídos na presente revisão. $\mathrm{Na}$ busca inicial realizada nas
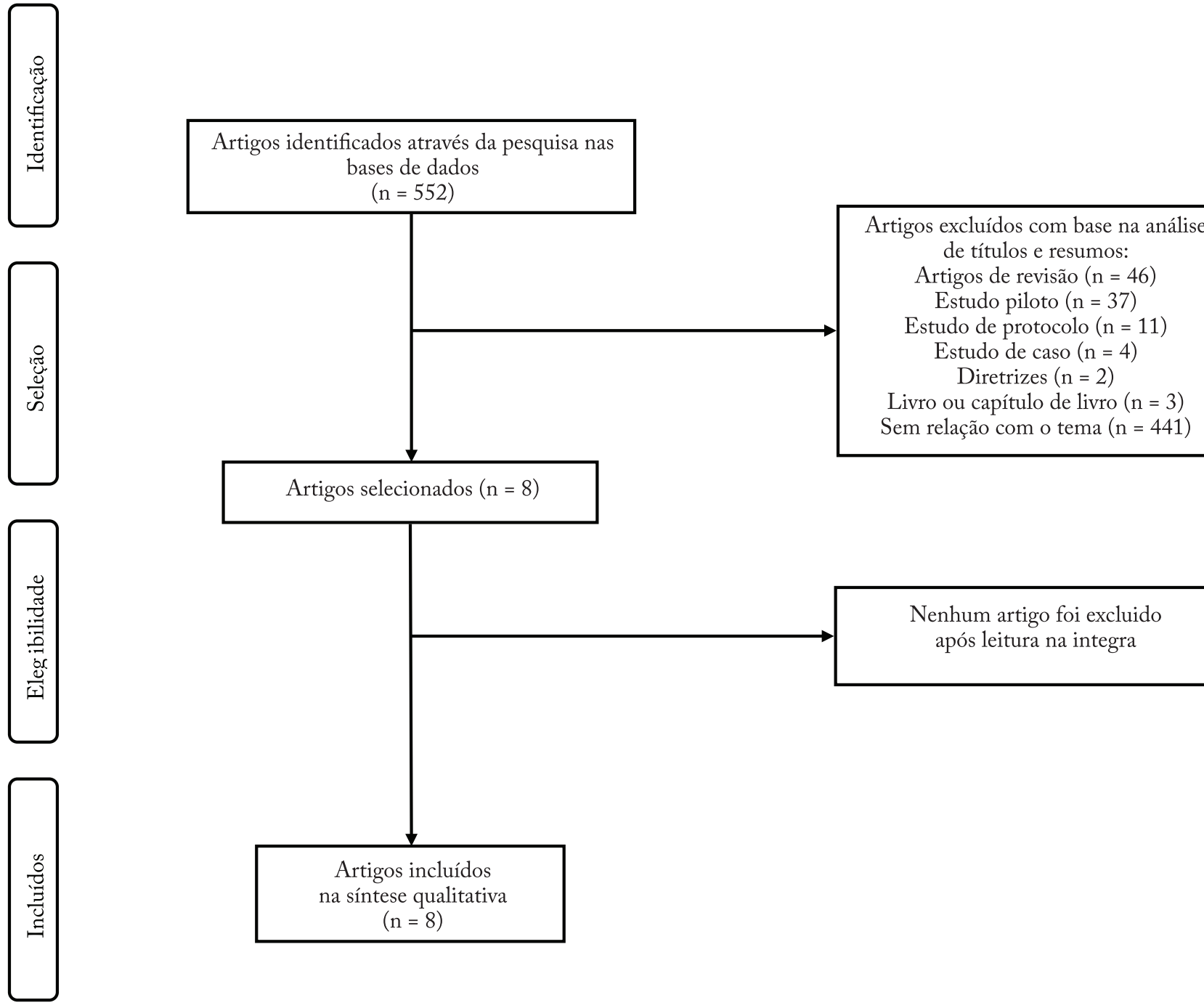

Nenhum artigo foi excluido após leitura na integra

Figura 2 - Fluxograma do estudo 
bases de dados foram encontrados 552 artigos, desses 103 foram excluídos por não serem estudos originais e 441 foram excluídos por não ter relação com o tema. Dessa forma, oito estudos foram utilizados nessa revisão sistemática.

$\mathrm{Na}$ tabela 1, estão apresentadas as características gerais dos estudos incluídos na revisão, a avaliação da qualidade de sono bem como as características dos programas de Pilates e os principais resultados dos estudos. Os anos de publicação dos estudos variaram de 2009 a 2018, eram adultos de ambos os sexos, sendo jovens universitários ${ }^{10}$, mulheres pós-parto ${ }^{11}$, mulheres pós-menopausa ${ }^{12}$, população sedentária ${ }^{13,14}$, idosas $^{16,19} \mathrm{e}$ diabéticos ${ }^{20}$, com idades de 18 a 65 anos, totalizando 608 participantes, sendo 303 participantes no grupo de Pilates. A avaliação da qualidade do sono na maior parte dos estudos foi obtida por meio de questionários: Pittsburgh Sleep Quality Index (87\% dos estudos) ${ }^{10-13,16,19,20} \mathrm{e}$ Escala de sonolência de Epworth ${ }^{14}$. Todos os estudos que informaram o método de Pilates, utilizaram o Mat Pilates (Pilates de Solo).

A maioria dos programas de treinamento foi realizado de 2 a 3 vezes na semana ${ }^{10,12-14,16,19}$ com uma duração média de 120 a 180 minutos de atividade por semana, de 13 a 15 exercícios, sendo de 1 a 2 series, 8 a 12 repetições, trabalhando o controle da respiração. A duração das intervenções variou de $4^{20}$ a $16^{16}$ semanas. Todos os estudos observaram melhoria na qualidade do sono geral no grupo de intervenção, sendo que os estudos de Ashrifinia et al. ${ }^{11}$, Ahmadinezhad et al. ${ }^{12} \mathrm{e}$ Aibar-Almazán et al. ${ }^{19}$ ainda reportam melhoria na latência do sono, disfunção diurna, percepção subjetiva de sono e redução do uso de medicamentos para dormir.

A Tabela 2 apresenta a pontuação da qualidade dos estudos de acordo com a análise da escala de PEDro. Os resultados revelaram que seis dos oitos estudos incluídos tiveram pontuação igual ou inferior a seis.

\section{Discussão}

O principal resultado encontrado neste estudo de revisão sistemática foi que a prática do Pilates através do método Mat Pilates com duração mínima de 4 semanas, duas a três sessões semanais, com um tempo médio de 120 a 180 minutos de atividade por semana, de 13 a 15 exercícios, de 1 a 2 series, com repetições de 8 a 12, trabalhando o controle da respiração foram suficientes para melhorar a qualidade do sono de adultos e idosos.

Um importante ponto a ser discutido é que, dentre os estudos analisados, apenas dois ${ }^{12,19}$ obtiveram uma pontuação maior que 6 em relação à escala PEDro, que visa avaliar os estudos em relação à validade interna e externa. Dentre os pontos avaliados, identificou-se que dois estudos deixaram explícito o cegamento dos avaliadores ${ }^{12,19}$, apenas dois estudos ${ }^{11,12}$ destacaram a intenção de tratar dos voluntários que não deram continuidade a intervenção e dois estudos não apresentaram grupo controle ${ }^{14,20}$, limitando sobremaneira a interpretação dos resultados e impactando fortemente na pontuação obtida. Assim, destaca-se a importância de os estudos reportarem informações associadas principalmente à validade interna da pesquisa.

Além disso, outro ponto que deve ser levado em consideração é o método exercício adotado nos estudos. A literatura reporta que o Pilates pode ser realizado de forma tradicional com equipamentos especializados onde a modulação da intensidade é realizada através dos aparelhos ou no solo, conhecido como Mat Pilates onde a modulação da intensidade se dá através dos exercícios posturais ${ }^{15}$. Na presente revisão apenas o Mat Pilates foi adotado como intervenção nos estudos incluídos.

Um dos aspectos que deve ser levado em consideração no momento da interpretação dos resultados é o instrumento utilizado para avaliar a qualidade do sono. Todos os estudos incluídos na revisão utilizaram apenas questionários para avaliar a qualidade do sono. $\mathrm{O}$ questionário PSQ ${ }^{21}$ avalia a qualidade do sono no último mês fornecendo um índice de severidade e natureza da desordem e a escala Epworth sleepiness scale (ESS) ${ }^{22}$ avalia o nível geral de sonolência diurna, ao avaliar o risco de propensão para dormir durante oito situações diurnas diferentes.

De maneira distinta, do que é recomendado, os estudos incluídos na presente revisão não avaliaram diretamente a qualidade do sono, (i.e., polissonografia). A polissonografia permite a avaliação do sono alterado durante todo o período noturno possibilitando o diagnóstico de grande parte dos transtornos do sono. Embora a avaliação subjetiva da qualidade do sono através do PSQI e ESS permita a monitorização das respostas aos tratamentos empregados conferindo aplicabilidade prática na rotina clínica ${ }^{23,24}$, seria interessante que estudos futuros analisassem o efeito do método Pilates na qualidade do sono através da polissonografia.

Não obstante o reconhecimento das limitações metodológicas empregadas nos diferentes estudos, os resultados do presente estudo demonstraram que o Mat Pilates foi eficaz na melhoria da qualidade do sono, e que do ponto de vista da saúde pública é interessante, dado sua relação direta entre a qualidade do sono, as 
Tabela 1 - Características gerais dos estudos, avaliação da qualidade de sono, característica do programa de Pilates e os principais resultados dos estudos incluídos na revisão.

\begin{tabular}{|c|c|c|c|c|c|c|c|c|}
\hline Autor/Ano & População & $\begin{array}{l}\text { Idade } \\
\text { (anos) }\end{array}$ & $\begin{array}{l}\text { Grupos } \\
\text { experimentais }\end{array}$ & $\mathrm{n}$ & $\begin{array}{l}\text { Avaliação } \\
\text { do sono }\end{array}$ & $\begin{array}{l}\text { Método } \\
\text { de Pilates }\end{array}$ & Programa de treinamento & Principais resultados \\
\hline $\begin{array}{l}\text { Caldwell et } \\
\text { al. }{ }^{10} \\
2009\end{array}$ & $\begin{array}{l}\text { Jovens } \\
\text { universitários }\end{array}$ & 18 a 32 & $\begin{array}{l}\text { Pilates } \\
\text { Tai Chi Chuan } \\
\text { Recreação }\end{array}$ & $\begin{array}{l}41 \\
29 \\
28\end{array}$ & PSQI & $\mathrm{NI}$ & $\begin{array}{l}15 \text { semanas sendo } 2-3 \text { vezes } \\
\text { semanais, totalizando } 150 \\
\mathrm{~min} / \mathrm{sem} \text {. }\end{array}$ & $\begin{array}{l}\text { Melhora na qualidade do } \\
\text { sono com redução no escore } \\
\text { do PSQI no grupo Pilates ( } \mathrm{p} \\
=0,002)\end{array}$ \\
\hline $\begin{array}{l}\text { Ashrafinia et } \\
\text { al. }{ }^{11} \\
2013\end{array}$ & $\begin{array}{l}\text { Mulheres } \\
\text { pós-parto }\end{array}$ & 18 a 35 & $\begin{array}{l}\text { Pilates } \\
\text { Controle }\end{array}$ & $\begin{array}{l}40 \\
40\end{array}$ & PSQI & Mat & $\begin{array}{l}8 \text { semanas sendo } 5 \text { vezes } \\
\text { semanais (home-based), } 13 \\
\text { exercícios, } 10 \text { repetições, } \\
\text { respiração profunda. Os } \\
\text { exercícios foram realizados no } \\
\text { início da manhã, } 30 \text { minutos } \\
\text { após a amamentação. }\end{array}$ & $\begin{array}{l}\text { Melhora na qualidade do } \\
\text { sono geral ( } p<0,001) \text { e na } \\
\text { percepção subjetiva de sono ( } p \\
<0,001) \text {, e redução na latência } \\
\text { do sono }(p<0,001) \text { e na } \\
\text { disfunção diurna }(p<0,001) \\
\text { apenas no grupo Pilates. }\end{array}$ \\
\hline $\begin{array}{l}\text { García-Soidán } \\
\text { et al. }{ }^{13} \\
2014\end{array}$ & $\begin{array}{l}\text { População } \\
\text { sedentária }\end{array}$ & 40 a 60 & $\begin{array}{l}\text { Pilates } \\
\text { Controle }\end{array}$ & $\begin{array}{l}48 \\
51\end{array}$ & PSQI & Mat & $\begin{array}{l}12 \text { semanas sendo } 2 \text { vezes } \\
\text { semanais, } 15 \text { exercícios de } \\
10 \text { repetições, controlando a } \\
\text { respiração, totalizando } 120 \\
\mathrm{~min} / \mathrm{sem} \text {. Os exercícios em }\end{array}$ & $\begin{array}{l}\text { Melhora na qualidade do sono } \\
\text { geral }(p<0,01) \text { e redução na } \\
\text { latência do sono }(p<0,01) \\
\text { apenas no grupo Pilates. }\end{array}$ \\
\hline
\end{tabular}

semanais, 2 series de 8 a

12 repetições, totalizando

$120 \mathrm{~min} / \mathrm{sem}$. Cada sessão

consistia em 3 fases: exercícios

de preparação (alongamentos), exercícios com bola e sem bola no solo e relaxamento.

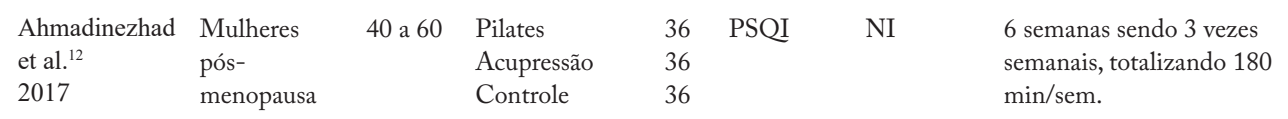

Melhora na qualidade do sono geral nos grupos Pilates e Acupressão ( $<$ 0,001). Além disso, melhora na percepção subjetiva de sono ( $p<0,001)$, aumento na eficiência do sono $(\mathrm{p}<0,001)$ e redução na latência do sono $(\mathrm{p}<0,001)$, na disfunção diurna ( $\mathrm{p}<$ 0,001) e no uso de medicação para dormir $(\mathrm{p}<0,001)$ no grupo Pilates.

\begin{tabular}{|c|c|c|c|c|c|c|c|c|}
\hline $\begin{array}{l}\text { Curi et al. }{ }^{16} \\
2018\end{array}$ & Idosas & $>60$ & $\begin{array}{l}\text { Pilates } \\
\text { Controle }\end{array}$ & $\begin{array}{l}31 \\
30\end{array}$ & PSQI & Mat & $\begin{array}{l}16 \text { semanas sendo } 2 \text { vezes } \\
\text { semanais, totalizando } 120 \\
\mathrm{~min} / \text { sem. }\end{array}$ & $\begin{array}{l}\text { Melhora na qualidade do sono } \\
\text { geral }(p=0,02) \text { e redução na } \\
\text { latência do sono }(p=0,02) \\
\text { e no uso de medicação para } \\
\text { dormir }(p=0,04) \text {. }\end{array}$ \\
\hline $\begin{array}{l}\text { Sharma et al. }{ }^{20} \\
2018\end{array}$ & Diabéticos & 35 a 65 & Pilates & 30 & PSQI & Mat & $\begin{array}{l}4 \text { semanas sendo } 5 \text { vezes } \\
\text { semanais, totalizando, } \\
30-40 \text { minutos cada sessão. } \\
\text { Exercícios de postura, de } \\
\text { respiração e controle muscular, } \\
\text { divididos em rotina básica, } \\
\text { intermediária e avançada. }\end{array}$ & $\begin{array}{l}\text { Melhora na qualidade do sono } \\
\text { geral } \\
(p<0,001) .\end{array}$ \\
\hline $\begin{array}{l}\text { Aibar- } \\
\text { Almazán et } \\
\text { al. }{ }^{19} \\
2019\end{array}$ & Idosas & $>60$ & $\begin{array}{l}\text { Pilates } \\
\text { Controle }\end{array}$ & $\begin{array}{l}55 \\
55\end{array}$ & PSQI & Mat & $\begin{array}{l}12 \text { semanas sendo } 2 \text { vezes } \\
\text { semanais, totalizando } 120 \\
\text { min/sem. Cada sessão } \\
\text { consistia em } 3 \text { fases: } 10 \\
\text { minutos de aquecimento, } \\
35 \text { minutos de exercícios de } \\
\text { Pilates e } 15 \text { minutos de volta } \\
\text { à calma. }\end{array}$ & $\begin{array}{l}\text { Melhora na qualidade do } \\
\text { sono geral ( } \mathrm{p}<0,001 \text { ) e na } \\
\text { percepção subjetiva de sono ( } \mathrm{p} \\
=0,001 \text { ) e redução na latência } \\
\text { do sono ( } \mathrm{p}<0,001) \text {, no uso de } \\
\text { medicação para dormir ( } \mathrm{p}< \\
0,001 \text { ) e na disfunção diurna } \\
(\mathrm{p}=0,008) \text { e aumento na } \\
\text { eficiência do sono ( } \mathrm{p}<0,001) \\
\text { apenas no grupo Pilates. }\end{array}$ \\
\hline
\end{tabular}

PSQI = Pittsburgh Sleep Quality Index; NI = Não informado; ESS = Escala de sonolência de Epworth; $\mathrm{n}=$ número de participantes 
Tabela 2 - Pontuação dos estudos de acordo com a escala de PEDro.

\begin{tabular}{|c|c|c|c|c|c|c|c|c|}
\hline & $\begin{array}{l}\text { Caldwell } \\
\text { et al. }{ }^{10}\end{array}$ & $\begin{array}{l}\text { Ashrafinia } \\
\text { et al. }{ }^{11}\end{array}$ & $\begin{array}{l}\text { García-Soidán } \\
\text { et al. }{ }^{13}\end{array}$ & $\begin{array}{l}\text { Leopoldino } \\
\text { et al. }{ }^{14}\end{array}$ & $\begin{array}{c}\text { Ahmadinezhad } \\
\text { et al. }{ }^{12}\end{array}$ & $\begin{array}{l}\text { Curi } \\
\text { et al. }{ }^{16}\end{array}$ & $\begin{array}{l}\text { Sharma } \\
\text { et al. }{ }^{20}\end{array}$ & $\begin{array}{c}\text { Aibar-Almazán } \\
\text { et al. }{ }^{19}\end{array}$ \\
\hline Elegibilidade & 0 & 1 & 1 & 1 & 1 & 1 & 1 & 1 \\
\hline Randomização dos grupos & 0 & 1 & 1 & 0 & 1 & 1 & 0 & 1 \\
\hline Alocação secreta & 0 & 0 & 0 & 0 & 1 & 0 & 0 & 1 \\
\hline Características iniciais similar & 1 & 1 & 1 & 0 & 0 & 1 & 0 & 1 \\
\hline Cegamento dos participantes & 0 & 0 & 0 & 0 & 0 & 0 & 0 & 0 \\
\hline Cegamento do treinador & 0 & 0 & 0 & 0 & 0 & 0 & 0 & 1 \\
\hline Cegamento dos avaliadores & 0 & 0 & 0 & 0 & 1 & 0 & 0 & 1 \\
\hline $\begin{array}{l}\text { Medida do desfecho em } 85 \% \text { dos } \\
\text { pacientes }\end{array}$ & 0 & 0 & 0 & 0 & 1 & 1 & 0 & 1 \\
\hline Intenção de tratar & 0 & 1 & 0 & 0 & 1 & 0 & 0 & 0 \\
\hline Diferença entre grupos & 1 & 1 & 1 & 0 & 1 & 1 & 0 & 1 \\
\hline $\begin{array}{l}\text { Medidas de tendência central e } \\
\text { variabilidade }\end{array}$ & 1 & 1 & 1 & 1 & 1 & 1 & 1 & 1 \\
\hline Pontuação total & 3 & 6 & 5 & 2 & 8 & 6 & 2 & 9 \\
\hline
\end{tabular}

doenças crônicas e eventos cardiovasculares ${ }^{3-5,25}$. Sabe-se que independentemente da idade, indivíduos com transtornos decorrentes do sono apresentam maior prevalência de fatores de risco cardiovascular que pessoas com boa qualidade do sono ${ }^{26,27}$.

No entanto, chama a atenção a escassez de estudos voltados para amostras exclusivas de idosos, em especial com doença cardiometabólicas, dado que esse grupo apresenta graves problemas relativos ao sono ${ }^{28,29}$. Caldwell et al. ${ }^{10}$ analisaram jovens universitários com idade entre 18 a 32 anos, enquanto Ashrafinia et al. ${ }^{11}$ apenas mulheres pós-parto, com idades semelhantes, entre 18 a 35 anos, se aproximando das idades observadas por Leopoldino et al. ${ }^{14}$ que foi de 18 a 30 anos. Nesses estudos, foram observadas além de melhora na qualidade geral do sono, reduções na latência do sono e na sonolência diurna. $\mathrm{O}$ único estudos em que a amostra foi composta exclusivamente por idosos for realizado por Curi et al. ${ }^{16}$, que submeteram idosos saudáveis (média de 64 anos) e diferentemente dos estudos anteriores, observaram além de melhora na qualidade geral do sono e redução na latência do sono, uma redução no uso de medicações para dormir. Por fim, apenas um estudo $^{20}$ foi composto de amostra com doença metabólica (Diabetes tipos II) porém com idade de 35 a 65 anos.

A baixa qualidade do sono é multifatorial. No entanto, fatores de risco como latência aumentada do sono, redução no tempo de sono, uso de medicações para dormir e distúrbios do sono ${ }^{30,31}$ afetam diretamente a percepção de qualidade do sono. Embora não tenha sido objetivo do presente estudo analisar os mecanismos pelos quais o Pilates promove melhoria da qualidade sono, sabe-se que ele atua diretamente no controle do estresse e na melhora da qualidade de vida $^{32-34}$, fatores relacionados com a qualidade do sono. Além do mais, entre os princípios básicos do método Pilates encontra-se a respiração diafragmática ${ }^{15}$. Essa condição faz com que o Pilates melhore a inspiração, que é um dos fatores associados a baixa qualidade do sono em adultos com apneia do sono ${ }^{35}$.

O presente estudo apresenta algumas implicações práticas, sobretudo para profissionais de saúde. Em resumo, os estudos com a realização do Mat Pilates adotaram protocolos entre 6 e 16 semanas de intervenção, com uma frequência de 2 a 3 vezes semanais, em média de 120 a 150 minutos de atividade por semana, de 13 a 15 exercícios, de 1 a 2 series, com repetições de 8 a 12 trabalhando o controle da respiração. No entanto, devido a heterogeneidade dos subgrupos populacionais analisados (jovens universitários, idosos, mulheres pós-menopausa e diabéticos) os achados apresentados devem ser verificados com cautela no momento da sua extrapolação, bem com as limitações metodológicas encontradas nos estudos. Portanto, futuros estudos com maior rigor metodológico são necessários para atestar a eficácia do Pilates na qualidade do sono.

A presente revisão tem limitações que devem ser consideradas. A pesquisa bibliográfica foi realizada apenas em periódicos indexados nas bases de dados eletrônicas PubMed/Medline, Lilacs, SciELO e SCOPUS. Dessa forma, é possível que alguns estudos sobre esta temática não tenham sido incluídos. Contudo, vale 
ressaltar que as bases de dados utilizadas no presente estudo são as mais consultadas para pesquisa bibliográfica de manuscritos em línguas portuguesa e inglesa. Além disso, a investigação foi realizada utilizando apenas descritores em inglês e português, portanto, estudos existentes em outros idiomas não foram incluídos. $\mathrm{E}$ por fim, os estudos incluídos adotaram apenas o Mat Pilates como método de exercício, o que não permite a comparação com outros métodos. Apesar disso, ao nosso conhecimento esse é o primeiro estudo de revisão sistemática que objetivou analisar os efeitos do Pilates na qualidade do sono de adultos e idosos e segundo, os procedimentos metodológicos adotados seguiram a recomendações PRISMA para estudos de revisão sistemática com revisão e extração dos dados por pares.

Em conclusão, a presente revisão sistemática, identificou que a realização do Pilates, em especial o de solo (Mat Pilates) com frequência mínima de duas vezes semanais e um mês melhora a qualidade do sono e reduz a sonolência diurna de adultos e idosos.

\section{Conflito de interesses}

Os autores declaram não haver conflito de interesses.

\section{Contribuição dos autores}

Lins-Filho OL, Oliveira LMFT, Queiroz GKF, Santos JFJ, Farah BQ, Santos MAM participaram da concepção inicial do estudo, redação e revisão crítica do texto. Queiroz GKF, Santos JFJ, Farah BQ foram responsáveis pela busca da literatura e coleta de dados.

\section{Referências}

1. Madrid-Valero JJ, Martinez-Selva JM, Couto BR, SanchezRomera JF, Ordonana JR. Age and gender effects on the prevalence of poor sleep quality in the adult population. Gac Sanit. 2017;31(1):18-22.

2. Silva CA, Lima WC. Exercício físico na melhora da qualidade de vida do indivíduo com insônia. Movimento. 2001;7(14):49-56.

3. Crispim CA, Zalcman I, Dáttilo M, Padilha HG, Tufik S, Mello MT. Relação entre sono e obesidade: uma revisão da literatura. Arq Bras Endocrinol Metab. 2007; 51(7):1041-49.

4. Pedrosa RP, Cabral MM, Pedrosa LC, Sobral Filho DC, Lorenzi-Filho G. Apneia do sono e hipertensão arterial sistêmica. Rev Bras Hipertens. 2009;16(3):174-177.

5. Hanus JS, Amboni G, Rosa MI, Ceretta LB, Tuon L. Características e qualidade do sono de pacientes hipertensos. Rev Esc Enferm USP. 2015;49(4):596-602.

6. Sengul YS, Ozalevli S, Oztura I, Itil O, Baklan B. The effect of exercise on obstructive sleep apnea: a randomized and controlled trial. Sleep Breath. 2011;15(1):49-56.

7. Steffens AK, Liz CM, Viana MS, Brandt R, Oliveira LG, Andrade A. Praticar caminhada melhora a qualidade do sono e os estados de humor em mulheres com síndrome da fibromialgia. Rev Dor. 2011;12(4):327-31.
8. Chang SP, Shih KS, Chi CP, Chang CM, Hwang KL, Chen YH. Association between exercise participation and quality of sleep and life among university students in Taiwan. Asia Pac J Public Health. 2016;28(4):356-67.

9. King AC, Oman RF, Brassington GS, Bliwise DL, Haskell WL. Moderate-intensity exercise and self-rated quality of sleep in older adults: a randomized controlled trial. JAMA. 1997;277(1):32-7.

10. Caldwell K, Harrison M, Adams M, Triplett NT. Effect of Pilates and Taiji Quan training on self-efficacy, sleep quality, mood, and physical performance of college students. J Bodyw Mov Ther. 2009;13(2):155-63.

11. Ashrafinia F, Mirmohammadali M, Rajabi H, Kazemnejad A, Sadeghniiathaghighi K, Amelvalizadeh M, et al. The effects of Pilates exercise on sleep quality in postpartum women. J Bodyw Mov Ther. 2014;18(2):190-9.

12. Ahmadinezhad M, Kargar M, Vizeshfar F, Hadianfard MJ. Comparison of the effect of acupressure and Pilatesbased exercises on sleep quality of postmenopausal women: a randomized controlled trial. Iran J Nurs Midwifery Res. 2017;22(2):140-6.

13. García-Soidán J, Giraldez VA, Zagalaz JC, Lara-Sánchez A. Does Pilates exercise increase physical activity, quality of life, latency, and sleep quantity in middle-aged people? Percept Mot Skills. 2014;119(3):838-50.

14. Leopoldino AA, Avelar NC, Passos GB, Santana NA, Teixeira VP, Lima VP, et al. Effect of Pilates on sleep quality and quality of life of sedentary population. J Bodyw Mov Ther. 2013;17(1):5-10.

15. Wells C, Kolt GS, Bialocerkowski A. Defining Pilates exercise: a systematic review. Complement Ther Med. 2012;20(4):253-62.

16. Curi VS, Vilaca J, Haas AN, Fernandes HM. Effects of 16-weeks of Pilates on health perception and sleep quality among elderly women. Arch Gerontol Geriatr. 2018;74:118-22.

17. Moher D, Liberati A, Tetzlaff J, Altman DG, Group P. Preferred reporting items for systematic reviews and meta-analyses: the PRISMA statement. PLoS Med. 2009;6(7):e1000097.

18. Maher CG, Sherrington C, Herbert RD, Moseley AM, Elkins M. Reliability of the PEDro scale for rating quality of randomized controlled trials. Phys Ther. 2003;83(8):713-21.

19. Aibar-Almazán A, Hita-Contreras F, Cruz-Diaz D, TorreCruz M, Jimenez-Garcia JD, Martinez-Amat A. Effects of Pilates training on sleep quality, anxiety, depression and fatigue in postmenopausal women: A randomized controlled trial. Maturitas. 2019;124:62-7.

20. Sharma D, Kaur J, Rani M, Bansal A, Malik M, Kulandaivelan S. Efficacy of Pilates based mat exercise on quality of life, quality of sleep and satisfaction with life in type 2 diabetes mellitus. Rom J Diabetes Nutr Metab Dis. 2018;25(2):149-56.

21. Buysse DJ, Reynolds CF, Monk TH, Berman SR, Kupfer DJ. The Pittsburgh Sleep Quality Index: a new instrument for psychiatric practice and research. Psychiatry Res. 1989;28(2):193-213.

22. Johns MW. A new method for measuring daytime sleepiness: the Epworth sleepiness scale. Sleep. 1991;14(6):540-5.

23. Santiago LC, Batista RM, Lyra MJ, Farah BQ, Pedrosa $\mathrm{RP}$, Falcao AP, et al. The role played by gender and age on poor sleep quality among institutionalized adolescents. Sleep Breath. 2017;21(1):197-202.

24. Chao LL, Abadjian LR, Esparza IL, Reeb R. Insomnia severity, subjective sleep quality, and risk for obstructive sleep apnea in veterans with Gulf war illness. Mil Med. 2016;181(9):1127-34. 
25. Oliveira BH, Yassuda MS, Cupertino AP, Neri AL. Relações entre padrão do sono, saúde percebida e variáveis socioeconômicas em uma amostra de idosos residentes na comunidade: Estudo PENSA. Cien Saude Colet. 2010;15(3):851-60.

26. Souza M, Medeiros AK, Carvalho MM, Medeiros CA, Lustosa TC, Couto TL, et al. Characteristics and predictors of obstructive sleep apnoea in patients with type 2 diabetes. Diabetes Metab. 2018;44(1):91-4.

27. Pedrosa RP, Drager LF, Gonzaga CC, Sousa MG, Paula LK, Amaro AC, et al. Obstructive sleep apnea: the most common secondary cause of hypertension associated with resistant hypertension. Hypertension. 2011;58(5):811-7.

28. Santos AA, Santos TC, Filomena CM, Iost SC. Sono, fragilidade e cognição: estudo multicêntrico com idosos brasileiros. Rev Bras Enferm. 2013;66(3): 351-57.

29. Chaput JP, Wong SL, Michaud I. Duration and quality of sleep among Canadians aged 18 to 79. Health Rep. 2017;28(9):28-33.

30. Lee W, Lee SA, Ryu HU, Chung YS, Kim WS. Quality of life in patients with obstructive sleep apnea: relationship with daytime sleepiness, sleep quality, depression, and apnea severity. Chron Respir Dis. 2016;13(1):33-9.
31. Claman DM, Ewing SK, Redline S, Ancoli-Israel S, Cauley JA, Stone KL, et al. Periodic leg movements are associated with reduced sleep quality in older men: the MrOS Sleep Study. J Clin Sleep Med. 2013;9(11):1109-17.

32. Vieira FT, Faria LM, Wittmann JI, Teixeira W, Nogueira LA. The influence of Pilates method in quality of life of practitioners. J Bodyw Mov Ther. 2013;17(4):483-7.

33. Oliveira LC, Oliveira RG, Pires-Oliveira DA. Effects of Pilates on muscle strength, postural balance and quality of life of older adults: a randomized, controlled, clinical trial. J Phys Ther Sci. 2015;27(3):871-6.

34. Pereira EC, Liberali R, Lopes CR, Cruz TM, Netto MI, Viana HB, et al. Aderência de pessoas no Pilates solo. Fisio Brasil. 2013;14(1):53-60.

35. Semelka M, Wilson J, Floyd R. Diagnosis and Treatment of Obstructive Sleep Apnea in Adults. Am Fam Physician. 2016;94(5):355-60. uma revisão sistemática. Rev Bras Ativ Fis Saúde. 2019;24:e0080. DOI: 10.12820/rbafs.24e0080. 